ihres hundertjährigen Bestehens noch ziemlich weit von Vollkommenheit. Den Beweis dafür finden wir in den von Zeit zu Zeit erscheinenden Vorschlägen, diese Methoden zu verbessern, sowie auch in Arbeiten, die in die diagnostische Methodik neue Symptome einzuführen oder die alten Behauptungen richtig zu stellen versuchen.

Es gehört hierher auch die Frage der Perkussions- und Auskultationssymptome bei pleuralen Flüssigkeitsansammlungen. An dieser Stelle wollen wir nur die neuen Beiträge zur perkutorischen Diagnostik der Pleuraergüsse berücksichtigen.

Fast alle Handbücher der Diagnostik geben als Regel an, daß die obere Dämpfungsgrenze bei pleuritischen Exsudaten in der Richtung von hinten nach vorne fällt, während sie bei Transsudaten horizontal verläuft. Dies soll vor allem von der Lage des Kranken, außerdem aber bei entzündlichen Ergüssen noch von den Verwachsungen abhängen, welche die Beweglichkeit der Flüssigkeit hemmen. Sahli, der erwähnt, daß auch bei geringen Transsudaten die obere Grenze gänzlich an die Exsudatlinie erinnern kann und daß anderseits in seltenen Fällen von Exsudaten die obere Grenze horizontal verlaufen kann, sieht die Ursache des Herabfallens der Linie nicht in der Schwerkraft, sondern in dem Verhältnis zwischen dem atmosphärischen Druck und der Retraktionskraft der Lunge. Zwar haben Damoiseau und Garland schon längst etwas anders die obere Grenze der Pleuraergüsse beschrieben, dies wird jedoch nur in gewissen Handbüchern als Illustration der Geschichte der Medizin angeführt. Nach Damoiseau nimmt die obere Flüssigkeitsgrenze eine elliptische oder parabolische Gestalt an, indem der Scheitelpunkt und die längere Achse dem hinteren Rande der Axillargrube entsprechen. Die beschriebene Form der Perkussionsgrenze soll lediglich bei der leisesten Perkussion festgestellt werden können. Diese Linie kann sich der Horizontalen nähern, falls die Flüssigkeit um $7-8 \mathrm{~cm}$ die Mamilla übersteigt. Gerland hat im Jahre 1878 eine Aufhellung des Perkussionsschalles neben der Wirbelsäule in Gestalt eines " $\mathrm{S}^{\text {" }}$ beschrieben, dessen Gipfel an der Axillarlinie und dessen unterer Punkt an der Wirbelsäule liegen. Aehnlich zeichnet diese Linie Ellis. Ritschi bestätigt die Beobachtung von Damoiseau klinisch und erwähnt dabei, dal das Atmungsgeräusch neben der Wirbelsäule tiefer als an anderen Stellen des Brustkorbs hörbar ist, was dafür spricht, daß hier die Flüssigkeitsmenge am geringsten ist.

In den letzten Jahren wurde wieder ein besonderes Augenmerk auf die Frage der Perkussionsdaten bei den-Pleuraergüssen, insbesondere bei den exsudativen gerichtet, wobei sich zwei. Fragen einstellten: manche Verfasser beobachteten gewisse Symptome an der gesunden, andere an der kranken Seite. Diese und jene Symptome sollten gewissermaben die Diagnose der Anwesenheit von Flüssigkeit im Pleuraraum erleichtern.

So notieren Grocco, Rauchfuss, Baduel und Siciliano, Korányi und andere bei den pleuritischen Exsudaten das Auftreten eines paravertebralen Dämpfungsdreiecks an der gesunden Seite, während Rosenbach, Kroenig und andere in diesen Fällen an der kranken Seite eine paravertebrale Aufhellungszone des Perkussionsschalles beschreiben. Hamburger hat schlieblich beide Symptome festgestellt. Es bestehen eigentlich gewisse Differenzen in den Details der Behauptungen einzelner Verfasser beider Gruppen, diese sind aber nicht besonders wichtig und verändern keinesfalls den Grund der Behauptung.

Rauchfuss spricht entschieden die Meinung aus: jeder freie, der Wirbelsäule anliegende Erguß, der bis zum achten Wirbel hinaufreicht, also noch vor Eintritt positiven Druckes in der Pleurahöhle, gibt einen deutlichen . . . . Dämpfungsbezirk auf der gesunden Seite ${ }^{\prime}$. Was die Spitze des Dreiecks anbetrifft, so entsprach dieselbe in der Hälfte von seinen Fällen ungefähr der Flüssigkeitshöhe, in den übrigen, besonders bei sehr abundanten Exsudaten, war sie um drei bis vier Wirbel tiefer. Dieses Dreieck soll nach Rauchfuss eine diagnostische Bedeutung haben, falls das Resultat der Probepunktion negativ ist, falls die Punktion aus gewissen Rücksichten unausführbar, oder wenn zu entscheiden ist, ob die auf der kranken Seite gebliebene Dämpfung noch durch die Flüssigkeitsanwesenheit oder durch andere Umstände verursacht ist. Es soll ferner die bei schwachen und fiebernden Kindern häufige

Perkussion und Auskultation - diese zwei Hauptgrund-

lagen der physikalischen Krankenuntersuchung - sind trotz

Aus der Inneren Abteilung des Krankenhauses Kindlein Jesu in Warschau. (Vorstand: Priv.-Doz. Dr. W. Janowski.)

\section{Ueber gewisse Perkussionssymptome bei} Pleuraergüssen.

Von Dr. Mieczyslaw Gantz. 
Atelektase ausschließen, und schließlich kommt dieses Symptom bei Lungenentzündung nicht zum Vorschein. Zwar ist auch bei dieser, bei stärkerer Perkussion, eine paravertebrale Dämpfungszone festzustellen, diese ist jedoch "bandförmig, diffus begrenzt " und niemals in Gestalt eines Dreiecks vorhanden. Im Gebiete des Dreiecks tritt nach Rauchfuss keine besondere Veränderung des Vesikuläratmens auf.

F. Korányi, welcher sich seinerzeit mit der Frage des allmählichen Auftretens der physikalischen Symptome bei den Pleuraexsudaten beschäftigte, beschreibt diese folgendermaßen: Die obere Dämpfungsgrenze soll entsprechend der Raschheit, mit der die Flüssigkeit zunimmt, gewisse Zeit schräg in der Richtung von der Wirbelsäule nach außen fallen, und erst mit der Zunahme des Exsudates sollen die Damois ea u schen Biegungen auftreten. Allmählich verbreitet sich die Dämpfung auch auf die gesunde Seite in Gestalt eines Dreiecks mit der Basis nach unten, was Resultat der Verschiebung des hinteren Mediastinums sein soll. Dieses Symptom hat Korányi (er will sich sogar die Priorität in dieser Frage vorbehalten) in mehr als $2 / 3$ der Fälle beobachtet. Dabei erwähnt er, daß die äußere Dämpfungslinie von oben und innen nach unten und außen in zahlreichen wellenartigen Biegungen verläuft.

Grocco hat ein ähnliches Dämpfungsdreieck im Jahre 1902 beschrieben, Baduel und Siciliano sollen sogar durch Versuche bewiesen haben, daß das Auftreten dieses Dreiecks in der Tat durch die Verdrängung des hinteren Mediastinums auf die gesunde Seite verursacht ist

Thayer und Fabyan haben ebenfalls ein Dämpfungsdreieck uf der gesunden Seite beobachtet, welches verschwand, sobald sich der Patient auf die kranke Seite legte. An dieser Stelle war das Atmungsgeräusch abgeschwächt oder bronchial, manchmal trat sogar A egophonie und "signe du sous“ auf.

Kraus stellte im Jahre 1905 in der Sitzung der Gesellschaft der Charitéärzte in Berlin Röntgenogramme vor, die er an Kranken mit Pleuraexsudaten erhalten hat. Auf den Röntgenogrammen soll nämlich eine Verdunkelung auf der gesunden Seite neben der Wirbelsäule auftreten, welche der paravertebralen Dämpfung entspricht. Dies entsteht, nach Kraus, infolge der Verschiebung des Mediastinums, und zwar fast ausschließlich der des vorderen. Was die obere Grenze des Exsudates anbetrifft, so verläuft sie nach Ansicht dieses Verfassers "gewöhnlich in einer von oben außen nach innen unten ziehenden Linie“. Man könnte daraus schließen, daß Kraus ebenfalls das Auftreten einer paravertebralen Aufhellung auf der kranken Seite annimmt.

Brudzinski, welcher diese Frage unlängst nachprüfte, fand in 18 Fällen von Pleuritis exsudativa bei $\mathrm{Kindern}$ bei allen die paravertebrale Aufhellung auf der kranken Seite, und dabei am häufigsten in Gestalt eines Dreiecks, und 17 mal außerdem die paravertebrale Dämpfung, ebenfalls dreieckiger Gestalt, auf der gesunden Seite. Die Größen beider Dreiecke waren meistenteils gleich. Die Dreiecke veränderten sich entsprechend dem Entleeren oder der Resorption des Exsudates. Im Gebiete des Dämpfungsdreiecks war das Atmungsgeräusch abgeschwächt, es kam auch „signe du sous“ zum Vorschein.

So kommen Grocco, Rauchfuss, Baduel und Siciliano, Kraus, Thayer und Fabyan, Hamburger, Brudzinski zu dem Schluß, daß bei pleuritischem Exsudat auf der gesunden Seite eine dreieckige paravertebrale Dämpfung mit der Basis nach unten auftritt. Diese soll nach der Meinung gewisser Verfasser durch die Verschiebung des Mediastinums bedingt sein, nach der Ansicht anderer spielt hier auch die größere Elastizität des Brustkorbs bei den Kindern eine Rolle, indem bei Pleuraergüssen die das Exsudat enthaltende Hälfte des Brustkorbs an der Perkussion der gesunden Seite keinen Anteil nimmt. Einige Verfasser (Ra u chfuss) finden schließlich keine Verănderungen des Atmungsgeräusches im Gebiete des Dämpfungsdreiecks, andere (Thayer und Fabyan, Brudzinski) stellen hier solche fest. Es ist noch hinzuzufügen, daß das besprochene Symptom von Rauchfuss, Brudzinski, Ham. burger bei Kindern beobachtet wurde.

Anders wird die Perkussionsfrage bei den Pleuraergüssen von Krönig, Rosenbach und Sokoloff erörtert. Die Untersuchungen von Sokol of $f$ haben für uns desto größeren Wert, da dieselben ebenfalls an Kindern unternommen und durch Röntgenogramme kontrolliert wurden. Diese drei Verfasser notieren lediglich eine Aufhellung des Perkussionsschalles neben der Wirbelsäule auf der kranken Seite mit dem Unterschied, daß Krönig geradezu als Regel“ in 1000 Fällen ein Dreieck beobachtete, Rosenbach

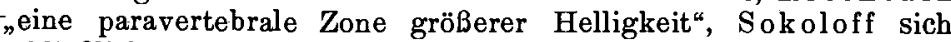
schließlich mit der Form beschäftigt, welche die Flüssigkeit in der Pleura annimmt und welche er als Pyramide beschreibt. Der letzte Verfasser wandte verschiedene Untersuchungsmethoden an; so leuchtete er alle Fälle durch, verglich die Durchleuchtungs- daten mit den klinischen Symptomen, kontrollierte durch Versuche die Untersuchungsergebnisse: a) mit Hilfe des in die Pleurahöhlen der Leichen eingegossenen Wassers bei vor- und nachheriger Röntgenographie, b) durch allmähliches Gefrieren der Leichen nach dem Eingießen, c) durch Eingießen von dickwerdenden Flüssigkeiten in die Pleurahöhlen, schließlich berücksichtigte er die anatomisch pathologischen Daten. Nirgends ist aber bei Sokoloff das Auftreten einer Dämpfung auf der gesunden Seite erwähnt, nirgends ist ferner an den Röntgenogrammen eine Verdunklung der gesunden Seite zu sehen, welche dem Dreieck von GroccoRauchfus s entsprechen sollte.

Seit gewisser Zeit haben wir auf der Abteilung von Herrn Priv.-Doz. Dr. Jan ow ski den Perkussionsdaten bei Pleuraergüssen eine besondere Aufmerksamkeit geschenkt und genau 30 Fälle $\left.^{1}\right)$ - 18 Exsudate, 12 Transsudate - beobachtet. Kein einziges Mal konnten wir, sogar bei der allerleisesten Perkussion, eine Dämpfung auf der gesunden Seite feststellen. Immer konnten wir dagegen folgendes finden:

1. der höchste Punkt der oberen Dämpfungslinie bei Exsudaten befindet sich nicht an der Wirbelsäule, sondern ist ziemlich weit von ihr nach auken entfernt und entspricht ungefähr dem Schulterblattwinkel;

2. die obere Dämpfungslinie fällt von diesem Punkte mehr oder weniger steil zur Wirbelsäule hinab, um mit ihr ein Dreieck mit der Basis nach oben zu bilden;

3. im Gebiete dieses Dreiecks ist der Perkussionsschall zweifelsohne heller als in der Gegend der Dämpfung, manchmal ist er sogar leicht tympanitisch;

4. da die obere Flüssigkeitsgrenze in der Richtung nach vorn und unten fällt, so nimmt der Erguß in der Tat die Form einer abgestumpften Pyramide an;

5. es gelang uns, dieselben Daten auch bei den pleuritischen Transsudaten zu beobachten.

Es ist hinzuzufügen, daß bei der zufälligen Durchleuchtung eines Falles mit pleuritischem Exsudat an dem Röntgenogramm eine deutliche paravertebrale Aufhellung des Schattens zu sehen war, welche die oben beschriebene Gestalt hatte. Schlieblich habe ich einmal beim Transsudat eine Probepunktion im Gebiete des Aufhellungsdreiecks mit negativem Erfolge ausgeführt, während sich bald nachher bei der Punktion auf derselben Höhe in der Gegend der Dämpfung die Flüssigkeit durch die Nadel unter ziemlich starkem Druck entleerte. Mit der Entleerung der Flüssigkeit wurde das Dreieck kleiner oder verschwand sogar vollkommen.

Es bleiben noch jetzt folgende Fragen zu beantworten:

1. Wie sind die von Sokoloff, Kroenig und uns erhaltenen Daten mit denen der Verfasser der ersten Gruppe zu vergleichen?

2. Wie ist die Entstehung des Aufhellungsdreiecks zu erklären?

Falls die Symptome von Grocco-Rauchfuss-Korányi lediglich bei Kindern zum Vorschein kämen, so könnte man diese Erscheinung ziemlich gut erklären (Brudzinski) durch die Theorie von Mazonn, nach dem die Entstehung des Perkussionsschalles nicht nur durch die Schwingungen der Luft in den Lungenalveolen (Skoda), sondern auch durch die des Brust. korbs bei der Perkussion (Williams) bedingt ist. Es ist klar, daß die Bedingungen in bezug auf den zweiten Punkt bei dem Pleuraergub und an gesunden Menschen verschieden. sind, sowie auch, daß dieser Punkt eine nicht geringe Bedeutung bei Kindern besitzen kann, bei welchen der elastischere Brustkorb in der Tat bei der allerleisesten (Rauchfuss) Perkussion der gesunden Seite auf die Wagschale fällt. Hamburger, der in einem Falle von Pleuraexsudat mit der Hand die gesunde Hälfte des Brustkorbes drückte, bekam die gewöhnlich auftretende paravertebrale Aufhellung auf der kranken Seite nicht mehr. Solche Umstände entsprechen vollkommen dem Vorschlag von Rauchfuss, dab man nämlich zum Zweck des Erhaltens der paravertebralen Dämpfung „die allerleiseste Perkussion" anwenden soll. Aehnliche Elastizität des Brustkorbs wie bei den Kindern besteht jedoch bei den Erwachsenen nicht, es kann also diese Ursache der Entstehung von paravertebraler Dämpfung auf der gesunden Seite bei den Er-

1) Inzwischen ist diese Zahl viel grőBer geworden. 
wachsenen, wenigstens in der Mehrzahl der Fälle, nicht in Betracht gezogen werden.

Die andere Erklärung dieses Symptoms, welche Rauchfuss, Grocco, Baduel und Siciliano annehmen, daß nämlich die erwähnte Dämpfung durch die Verdrängung des Mediastinums (des hinteren) verursacht ist, findet keine Bestätigung in den ausführlichen Versuchen von Sokoloff.

Was die Entstehung des paravertebralen Aufhellungsdreiecks auf der kranken Seite anbetrifft, so ist diese zweifelsohne durch die verschiedene Retraktionskraft der einzelnen Lungenteile bedingt. Kroenig meint, daß das Auftreten der in Frage stehenden Aufhellung des Perkussionsschalles einfach durch die anatomischen Verhältnisse bedingt ist. Da sich nämlich der Stiel der Lunge, d. i. ihr Punctum fixum gleich neben der Wirbelsäule befindet, so muß sich in dieser Richtung die Lunge beim Vorhandensein des Pleuraergusses zusammenziehen (falls, selbstverständlich, die Bewegungsfähigkeit der Lunge durch die eventuell bestehenden Verwarhsungen nicht beeinträchtigt wird). An dieser Stelle ist infolge des natürlichen Mangels an freiem Raum entweder keine oder nur eine geringe Menge von Flüssigkeit vorhanden. Wenn wir aber einen sehr abundanten Ergub und dementsprechend eine stärkere Retraktion oder Kompression der Lungen annehmen werden, so wird auch in diesem Falle der Perkussionsschall an dieser Stelle sogar durch eine beträchtlichere Flüssigkeits. schicht bei offenen Bronchien deutlich tympanitisch sein und sich ziemlich stark von dem Schenkelton der Nachbarschaft unterscheiden (Kroenig).

Resümee. 1. Der höchste Punkt der oberen Dämpfungslinie bei Pleuraergüssen befindet sich nicht an der Wirbelsäule, sondern entspricht ungefähr dem Schulterblattwinkel.

2. Von diesem höchsten Punkte fällt die obere Dämpfungslinie ziemlich steil zur Wirbelsäule, mit welcher sie ein Dreieck mit der Basis nach oben bildet, und sanfter nach vorne hinab.

3. Im Gebiete dieses Dreiecks ist der Perkussionsschall heller als in der Gegend der Dämpfung, manchmal sogar tympanitisch.

Literatur. 1. J. Brudzinski, Ueber das Symptom von Grocco-Rauchfuss-Hamburger bei Pleuritis bei Kindern (poln.). Tyg. Lek. 1908, No. 8. 2. Ko rả nyi, Ueber den Perkussionsschall der Wirbelsäule und dessen diagnostische Verwertung. Zeitschrift für klinische Medizin 1906, Bd. 60, H. 3 u. 4. - 3. K ra us Deutsche medizinische Wochenschrift 1905, No. 40, Gesellschaft der Charitè-Aerzte 4. Kroenig, Ueber das Verhalten des medialen Abschnittes der hinteren oberen Dampfungsgrenze bei pleuralen Flassigkeltsansammlungen. Berliner klnische Wochen schrift 1906, No. 13. - 5. v. Le ube, Diagnose der inneren Krankheiten 1902. Bd. 1. 6. Rauchfuss, Ueber die paravertebrale Dämpfung auf der gesunden Seite be Pleuraergüssen. Deutsches Archiv für klinische Medizin, Bd. 89, H. 1-4. - 7. Ro s e nbach, Die paravertebrale Aufhellungszone bei pleuritischen Exsudaten. Berliner klinische Wochenschrift 1906, No. 15. - 8. Sahli, Lehrbuch der klinischen Untersuchungsmethoden 1898,1905 . 